\title{
Terminal
}

Technologie de l'information, culture \& société

118 | 2016

Contrôle social, surveillance et dispositifs numériques

\section{La loi sur le renseignement}

Interview de Laurence Blisson, secrétaire générale du Syndicat de la

Magistrature

\section{Dominique Desbois et Jacques Vétois}

\section{Qrevues.org}

\section{Éditeur}

CREIS-Terminal

Édition électronique

URL : http://terminal.revues.org/1394

DOI : $10.4000 /$ terminal. 1394

ISSN : 2429-4578

Ce document vous est offert par Institut des sciences et industries du vivant et de l'environnement - AgroParisTech

\section{AgroParisTech}

Référence électronique

Dominique Desbois et Jacques Vétois, «La loi sur le renseignement », Terminal [En ligne], 118| 2016, mis en ligne le 15 septembre 2016, consulté le 23 mai 2017. URL : http://terminal.revues.org/1394 ; DOI : $10.4000 /$ terminal. 1394

Ce document a été généré automatiquement le 23 mai 2017.

tous droits réservés 


\title{
La loi sur le renseignement
}

\author{
Interview de Laurence Blisson, secrétaire générale du Syndicat de la \\ Magistrature
}

\section{Dominique Desbois et Jacques Vétois}

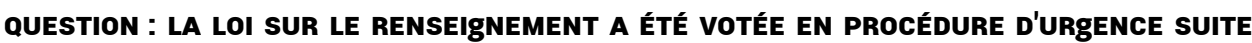
AUX ATTENTATS DU 11 JANVIER 2015. CETTE LOI VIENT S'AJOUTER À LA VINgTAINE DE LOIS SUR LA SÉCURITÉ DÉJÀ ADOPTÉES PAR LES gOUVERNEMENTS SUCCESSIFS DEPUIS LE DÉBUT DU SIÈCLE. EN QUOI CE CHOIX EST-IL CONTESTABLE ?
\end{abstract}

La loi est adoptée après les attentats de janvier 2015 qui ont été invoqués pour justifier cette procédure d'urgence alors que le texte était en préparation de longue date et que l'on en discernait les orientations dans un certain nombre de rapports parlementaires. En particulier, le rapport Cavard - Urvoas ${ }^{1}$ de 2013 dessinait très clairement les dispositions de ce texte. Utilisée comme procédure d'examen parlementaire, l'urgence invoquée pose problème par ce qu'elle produit: un débat réduit dans le temps car circonscrit à quelques journées en réalité pour chaque assemblée. Les consultations auprès des associations ont été menées dans la précipitation la plus totale alors que nous avions un texte doublement complexe à la fois au plan des techniques de renseignement mobilisées mais aussi au plan politique, comme première loi qui véritablement élaborait un cadre juridique des activités de renseignement. Son examen ne pouvait se faire dans un contexte aussi peu propice au questionnement sur le contenu. Ce qu'a produit l'urgence, on l'a entendu dans la bouche de certains parlementaires qui s'en sont remis à des experts. En particulier, certains parlementaires portaient le texte sans pouvoir en appréhender ni les conséquences constitutionnelles, ni même les techniques utilisées (par exemple ce qui relevait de la notion d'algorithme). L'urgence a donc permis d'imposer un texte qui aurait mérité un travail parlementaire plus important.

Ce recours à la procédure d'urgence n'est pas nouveau. Dans le domaine de la surveillance et dans celui des dispositifs antiterroristes, le recours à la procédure d'urgence est quasi systématique ; il a toujours produit une sorte de consensus, imposé 
par une incapacité à penser en dehors du cadre fixé, sur des textes qui ont rarement été soumis à un contrôle de constitutionnalité.

\section{QUESTION : SUR LE FOND, QU'EST-CE QUE CETTE LOI APPORTE PAR RAPPORT AUX AUTRES Mesures mises en œuVRe précédemment du point de VUe de la sécurité, du CONTRÔLE ET DES gaRANTIES POUR LES LIBERTÉS PUBLIQUES.}

Les lois précédentes en matière de terrorisme se focalisaient beaucoup sur la question de la répression et sur la création d'une architecture institutionnelle répressive extrêmement musclée, avec une centralisation des procédures dans les mains de certains juges, des pouvoirs d'enquête toujours plus avancés, et une répression toujours plus forte. Des infractions spécifiques ont contribué à pénaliser au stade de l'intention : c'est le rôle joué par l'infraction - centrale dans les poursuites - d'association de malfaiteurs à visée terroriste qui a trouvé avec la loi du 13 novembre 2014 des extensions dans le délit d'entreprise individuelle terroriste. On assiste à un accroissement des pouvoirs répressifs de l'État et des formes de surveillance au nom de la lutte contre le terrorisme.

Dans ce contexte, intervient une loi sur le renseignement ayant un objet bien plus large: selon le gouvernement, elle doit encadrer l'activité des services de renseignements.

Le renseignement, c'est surveiller pour identifier des menaces potentielles aux intérêts de l'État. Et surveiller qui? Surveiller les citoyens, les personnes se trouvant sur le territoire national, ou se trouvant à l'étranger. La notion d'intérêt de l'État va être un des nœuds du problème. Au nom de ce droit fondamental qu'est la protection des citoyens contre le risque d'arbitraire de l'État, on perçoit bien la nécessité évidente d'encadrer les mesures de surveillance mises en place par l'État. Dans le cadre de cette loi sur le renseignement, on réalise assez vite que ce texte ne crée pas les conditions de ce droit à la sûreté. Pour trois raisons que nous avons dénoncées :

- le champ d'intervention de cette loi ;

- les techniques de surveillance qui sont utilisées et légalisées;

- l'absence de contrôle indépendant et effectif de l'usage des techniques de renseignement.

En raison de ces trois failles, nous affirmons que le gouvernement n'a pas véritablement créé de cadre : encadrer aurait signifié fixer des limites aux services de renseignements mais ce que la loi produit c'est rendre légales des activités jusqu'à présent illégales. Invoquant un argumentaire un peu spécieux pour créer une catégorie "entre deux » d'actes "a-légaux ", l'activité des services de renseignements relèverait d'une "zone grise »: «l'a-légalité». En matière de légalité, le "a» privatif est une notion assez étonnante pour un juriste !

\section{QUESTION : MAIS CETTE ZONE gRISE A TOUJOURS EXISTÉ ?}

Ces actes illégaux n'étaient jamais réprimés par les tribunaux: cependant, on a un exemple en la matière qui concerne Bernard Squarcini ${ }^{2}$. En dehors de ce cas, on n'a pas connaissance de condamnation des services de renseignements pour l'usage d'un certain nombre de techniques intrusives sans base légale. Le problème, c'est que la loi sur le renseignement autorise tout dans un champ particulièrement large sans le contrôle nécessaire par une autorité indépendante.

Sur la question du champ d'abord, pour détailler ces trois caractéristiques principales qu'on contestait dans la loi, les débats ont porté sur ce qui relève des intérêts permettant de recourir aux techniques de renseignement. L'activité du renseignement, 
il faut le rappeler, est purement préventive. Et c'est une activité dans laquelle les services exercent une surveillance pour détecter une potentialité. Cet objet du renseignement diffère ainsi par la nature des pouvoirs de surveillance judiciaire qui ont pour but la constatation d'une infraction précise ou à tout le moins déterminable, qu'elle se soit produite ou qu'elle soit sur le point de se produire. Le renseignement se situe bien en amont de tout cela: on est toujours au conditionnel quant on parle de renseignement. La loi autorise les services de renseignements qui fonctionnent dans le secret à recourir à des techniques pour un certain nombre de motifs relatifs à la défense et à la promotion des intérêts fondamentaux de la nation. Il y a là une conception qui n'est pas seulement défensive (se prémunir contre une menace) mais qui est aussi offensive car on parle de la promotion des intérêts de la nation. Au nombre des items listés à l'article L811-3 du code de la sécurité intérieure, donc le nouvel article, les techniques de renseignement peuvent être utilisées pour la défense et la promotion de la politique étrangère mais aussi des intérêts de la politique économique, industrielle et scientifique de la France. À travers ces deux items ayant trait à la politique étrangère et économique de l'État mais aussi des entreprises nationales, on perçoit que la surveillance envisagée va bien au delà de la lutte contre le terrorisme pour toucher des personnes ou des organisations qui, parce qu'elles dénoncent des pratiques amorales dans la politique étrangère ou économique, vont à l'encontre des intérêts de l'État: dans cette situation, les intérêts de l'État ne s'assimilent pas aux intérêts des citoyens. Si on prend l'exemple d'une firme pharmaceutique ayant des liens avec une autre firme qui aurait des contrats avec l'État dans le domaine de l'armement ou dans le domaine de l'énergie et qui développerait des produits nocifs, la dénonciation par certains de ces pratiques pourrait être contraire à la promotion des intérêts de la firme.

Autre critère très problématique permettant le recours à ces techniques de surveillance, c'est la prévention des violences collectives qui peuvent porter atteinte gravement à la paix publique. D'après ce qu'en disent le Conseil constitutionnel et les parlementaires lors de la discussion de la loi, derrière cette notion d'atteinte grave à la paix publique, se profile la possibilité pour les services de renseignements d'opérer des mesures de surveillance vis à vis de personnes engagées dans le mouvement social, vis à vis d'organisations politiques ou syndicales ou de militants, affiliés ou non, dès lors qu'ils seraient susceptibles d'organiser ou de vouloir prendre part à des manifestations porteuses en leur sein de violences potentielles. Le flou et la conception très étendue de la notion de violences collectives et d'atteinte à la paix publique créent les conditions d'une surveillance qui va bien au delà de la notion de prévention du terrorisme. Cette notion ne doit par ailleurs pas être laissée de côté comme n'étant susceptible d'aucune interrogation: on sait qu'en droit français la définition des actes de terrorisme est particulièrement large car elle ne se borne pas aux actes portant atteinte à l'intégrité physique ou à la vie humaine mais inclut les infractions aux biens que sont les dégradations, le vol, le recel. Historiquement et encore récemment, si on repense à l'affaire de Tarnac par exemple, la définition de ce qui relève du terrorisme ou ce qui n'en est pas, a maille à partir avec des considérations politiques sur l'identification de groupes, en l'occurrence la gauche radicale comme relevant d'une activité terroriste ou d'une activité de contestation de « droit commun ».

La deuxième difficulté contestée, c'est la dimension particulièrement intrusive des pouvoirs qui ont été donnés aux services de renseignements puisque ce texte prolonge le texte existant sur les interceptions de sécurité, écoutes administratives effectuées 
par ces services. Il les prolonge puisqu'il permet la mise sous écoute administrative de l'entourage des personnes « ciblées » qu'elles aient une participation active ou non à l'activité suspectée, qu'elles aient une action consciente ou non dans cette activité, conduisant Jean-Marie Delarue, président de la Commission Nationale Consultative des Interceptions de Sécurité (CNCIS) ${ }^{3}$ à s'émouvoir très fortement de cette possibilité. Le texte donne des pouvoirs exorbitants en situation d'urgence: les services de renseignements peuvent directement ponctionner les données de connexion sur les réseaux, capter l'activité (image et son) d'un ordinateur en temps réel et recourir à des « sonorisations» (pose de micros à domicile). Dans certains lieux, ils peuvent installer des Imsi-Catchers ${ }^{4}$ qui servent à collecter massivement des données de connexion mais peuvent aussi brouiller les communications et capter les contenus. Le débat n'a malheureusement pas abouti au rejet de cette possibilité par les parlementaires alors que l'on pouvait espérer que ce qui relève clairement de la surveillance de masse soit supprimé du texte. La disposition la plus emblématique de cette surveillance de masse est la surveillance algorithmique du Net: sur la base de cette loi, les services peuvent appliquer des algorithmes sélectionnant des sondes installées sur l'ensemble des réseaux pour détecter des activités définies comme suspectes.

Dans l'ensemble de ces techniques de surveillance, les prétendues garanties qui tiendraient à une soit disant anonymisation - au motif que seules les métadonnées seraient concernées, que les algorithmes seraient définis de manière restreinte - n'en sont pas réellement: l'histoire de la contestation des mesures de surveillance nous le confirme sur l'ensemble de ces questions. On dispose de techniques particulièrement intrusives et en corollaire, il n'y a pas de réel contrôle indépendant. La Commission Nationale de Contrôle des Techniques de Renseignement (CNCTR) créée par cette loi est bien mal nommée : sa dénomination contient le mot contrôle alors qu'elle n'en exerce aucun puisqu'elle est une simple autorité consultative. Le Premier ministre, donc l'exécutif, reste l'autorité décisionnaire en la matière. Dans tous les domaines le Premier ministre peut passer outre l'avis de la CNCTR: car ce n'est pas un avis conforme qui est requis et, en cas d'urgence, son avis n'est même pas sollicité a priori. Le Syndicat de la Magistrature (SM) a revendiqué que cette autorité soit composée de juges administratifs et judiciaires et devienne une véritable autorité de décision: l'exécutif devrait solliciter une autorisation pour effectuer un acte de surveillance et cette autorité indépendante autoriserait ou pas la mise en œuvre de ces mesures par les services de renseignements. Le contrôle a priori et donc systématique est le seul cadre permettant d'assurer un contrôle effectif. C'est tout l'inverse de ce que prévoit le texte de loi : le Premier ministre adresse la demande à la CNCTR qui donne un avis et il peut passer outre. Ce n'est pas un cas d'école : du temps de la CNCIS, on a vu que Manuel Valls avait passé outre les précédents avis. S'il passe outre, la CNTCR peut faire une recommandation puis saisir le Conseil d'État, lequel statuera sur la légalité de la mesure de surveillance. Sachant que le Conseil d'État peut être saisi soit par la CNCTR, soit par un citoyen qui se penserait surveillé, pourquoi estimons-nous que ce contrôle par le Conseil d'État est insuffisant et qu'en réalité il est largement illusoire ? Le contrôle par la saisine du Conseil d'État par un citoyen est illusoire : d'abord du fait du caractère logiquement - secret de la mesure, puis des obstacles qui ont trait à la procédure devant le Conseil d'État qui est placée quasi systématiquement sous le sceau du secret-Défense, et enfin de l'absence de débat contradictoire ou de sa tenue tellement aménagée que la 
contradiction n'existe plus vraiment. Alors que l'exigence d'un débat contradictoire est un principe fondamental du droit à un procès équitable.

QUESTION : LA CNCTR DEVRAIT ÊTRE UNE AUTORITÉ INDÉPENDANTE ? POURTANT, CELLES-CI NE BRILLENT PAS EN FRANCE PAR LEUR INDÉPENDANCE NI LEUR ESPRIT CRITIQUE.

Le terme d'autorité indépendante n'est pas adapté: nous ne voulions pas d'une juridiction qui soit composée de parlementaires pour plusieurs raisons : pour assurer la véritable indépendance de cette institution de contrôle, elle ne peut dépendre ni de l'exécutif, ni du législatif. Le rôle du législatif est de surveiller l'exécutif dans le cadre des commissions d'enquête parlementaire et de son travail d'élaboration de la loi. Si on retire à l'exécutif le pouvoir de décider de mesures individuelles, il n'y a pas de raison d'intégrer le pouvoir législatif à la CNCTR comme moyen d'assurer l'équilibre des pouvoirs contre une utilisation dévoyée des moyens de surveillance à des fins politiques. Les autorités administratives n'ont pas pris place dans l'architecture institutionnelle parce qu'elles ne sont pas décisionnaires.

La création d'une juridiction indépendante décisionnelle aurait fait la différence, cependant c'est une condition nécessaire mais non suffisante. Il fallait mettre en place une structure effectuant un contrôle systématique et a priori mais aussi définir d'une manière restrictive les techniques et les champs d'intervention dans la loi. Ce n'est qu'à ces conditions-là que l'on peut espérer avoir un contrôle effectif.

QUestion : c'est là que se pose le problème du contrôle de la juridiction.

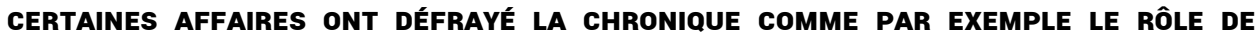

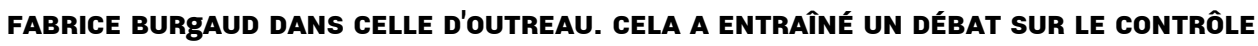
DES JUges d'INSTRUCTION. L'OPÉrATION "MAINS PROPRES " EN ITALIE A Également SUSCITÉ LA CRAINTE D'UN « gOUVERNEMENT DES JUgES ».

Selon le gouvernement au cours de ces débats, la question du renseignement relève d'une prérogative de l'exécutif et l'exécutif est responsable d'une certaine manière devant la représentation parlementaire (même si cela n'est pas si clair avec le Premier ministre). Le gouvernement affirmait que cela devait rester entre les mains de l'exécutif et non faire l'objet d'un contrôle par les juges. C'est un argument réversible : en matière de justice, le gouvernement définit des priorités mais les mesures individuelles relèvent de l'autorité judiciaire indépendante car l'atteinte aux libertés individuelles relève de la mission constitutionnelle de la justice. Sur la question de la responsabilité qui pourrait découler d'un mésusage du droit par une autorité juridictionnelle, il y a un mécanisme de responsabilité des juges et des voies procédurales existantes qui peuvent être mis en œuvre: cela s'applique de la même manière pour une autorité juridictionnelle qui serait constituée. C'est un sujet qu'il ne faut pas éluder mais qui ne saurait conduire à accepter que des mesures aussi intrusives soient placées entre les mains de l'exécutif.

QUESTION : ON NE PEUT PAS NIER QU'IL FAILLE DES SERVICES SECRETS. IL FAUT SURVEILLER LES ACTIONS DES gROUPES TERRORISTES, DES PUISSANCES ÉTRANgères ET MÊME LES

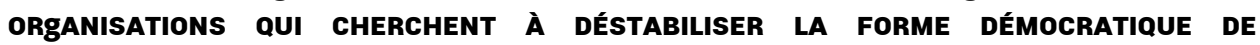
gOUVERNEMENT ; EST-CE QUE L'ON PEUT AVOIR UN MINIMUM DE gARANTIES QUE L'ACTION DES SERVICES SECRETS RESTERA DANS UN CADRE BIEN DÉfINI?

Le fonctionnement des services secrets est un domaine pour lequel la question de la règle qui n'a pas existé pendant des années (et celle de son éventuelle violation) n'est pas étrangère. Il faut penser l'élaboration d'un texte de loi en la matière comme une nécessité. Comment ensuite définir un cadre pertinent et démocratiquement acceptable 
pour l'activité de ces services ? Il y a la question du contrôle, déjà évoquée, et celle des techniques et du champ d'intervention. Il faut pouvoir définir ce qui relève légitimement de la surveillance; par exemple si on prend les critères qui sont ceux de la loi sur les intérêts de la politique étrangère, on peut estimer que les risques d'atteinte à l'indépendance nationale ou à l'intégrité du territoire sont des motifs légitimes de mise en œuvre de l'action des services de renseignements.

Il y a un principe général qui est de refuser le « renversement » de la surveillance : elle peut avoir sa légitimité dans certains domaines restreints si elle porte sur une personne en raison d'éléments objectifs qui laissent suspecter qu'elle rentre dans le cadre fixé. Elle devient totalement illégitime quand on opère une surveillance générale pour détecter les personnes qui seraient suspectes. On doit partir des éléments concernant une personne (cela peut conduire à obtenir des informations sur d'autres personnes) et non pas à l'inverse partir d'une surveillance générale pour cibler des personnes. C'est la règle de base qui fait qu'un certain nombre de dispositifs prévus dans la loi ne sont pas acceptables : les algorithmes, les imsi-catcher, les écoutes de l'entourage.

Et puis, dans un domaine qui ne relève pas du cadre des infractions pénales précisément définies par la loi, il faut respecter un principe de stricte nécessité offrant une protection forte contre les atteintes à la vie privée. Par exemple, nous avons soutenu que la sonorisation d'un domicile ne saurait être possible dans le domaine du renseignement. S'il y a suspicion d'une infraction pénale précise ou au moins déterminable, on rentre alors dans un cadre judiciaire, c'est à dire procédural et contradictoire, à un moment de la procédure qui peut rendre acceptable sous certaines circonstances l'utilisation de ces techniques intrusives, mais pas au stade du renseignement.

Sur la question des objectifs qui peuvent justifier le recours à des mesures de surveillance, la politique étrangère ne peut à elle seule le justifier. L'ingérence étrangère doit être perçue comme un risque direct de déstabilisation, précis et déjà identifié qui ne saurait justifier une écoute internationale massive.

Concernant les intérêts économiques, industriels et scientifiques, nous nous sommes posés la question de la lutte contre l'espionnage économique: il s'agit de prévenir l'espionnage dans le respect du droit à l'information. Dans le domaine économique, les éventuelles mesures de surveillance devraient être parfaitement encadrées car on perçoit bien les risques de dérives : cette éventualité a été utilisée pour bâillonner un certain nombre de lanceurs d'alertes. Pour tout ce qui concerne la prévention du terrorisme, de la délinquance et de la criminalité organisée, la difficulté sera de définir la barrière entre ce qui relève de la surveillance administrative préventive et ce qui relève d'une enquête judiciaire impliquant une affaire pénale ou une suspicion d'infraction pénale commise ou en préparation. Il faut que cette infraction soit déterminable. Dans le domaine judiciaire, nous disposons d'un véritable contrôle indépendant. Le risque est donc que les services procèdent à un contournement des réglementations en recourant aux surveillances administratives dans un cadre où en réalité on est en présence d'une infraction pénale. C'est difficile d'élaborer comme ça en quelques phrases un système de surveillance acceptable mais, en toute hypothèse, il se lit en creux dans tout ce que nous rejetons et ce que nous souhaitons élargir comme cadre juridique et ajouter comme contrôle juridictionnel. 


\begin{abstract}
QUESTION: VOUS FAITES LE CONSTAT DE L'INCOMPÉTENCE JURIDICTIONNELLE ET TECHNIQUe des pOLITIQUES. POURQUOI LES POLITIQUES N'ÉCOUTENT-ILS PAS LES MAgISTRATS DANS L'ÉLABORATION DES LOIS ET DE LA LOI SUR LE RENSEIgNEMENT EN PARTICULIER ?
\end{abstract}

Ce qui manque, c'est la volonté d'établir un système compatible avec notre système institutionnel et notre fonctionnement démocratique. Ceux qui portent ce texte ne font preuve ni d'incompétence, ni d'inconséquence. Ils savent ce qu'ils font. Ils veulent que l'exécutif conserve et développe ces capacités d'action en la matière et ils savent ce qu'ils ne veulent pas. Si on lit les rapports parlementaires de 2013, il y avait une volonté d'écarter par principe le contrôle juridictionnel du juge judiciaire dans un souci d'urgence et d'efficacité.

Sur la question de l'urgence, l'activité judiciaire connait bien cette notion et l'applique notamment pour autoriser des actes intrusifs : il est faux de prétendre que l'autorité judiciaire ne serait pas capable d'intervenir en urgence. Par contre, ce qui est inacceptable, c'est que l'urgence devienne un prétexte à l'absence de contrôle. Le contrôle est possible même dans l'urgence. Quand un juge d'instruction est sollicité par les policiers pour mettre une personne sur écoute (ils ne peuvent le faire d'eux-mêmes), le juge d'instruction va réagir dans les heures qui suivent puisque c'est une question particulièrement urgente. Il va néanmoins exercer un contrôle et s'assurer que l'écoute est nécessaire, proportionnée et qu'elle entre dans le cadre de la loi. Qu'est-ce qui fait que dans le cadre du renseignement, un tel contrôle ne soit pas possible? C'est une pure affirmation de l'exécutif que cela ne puisse l'être. En réalité, la question de l'urgence est une fausse problématique destinée à discréditer par principe le contrôle.

La question de l'efficacité n'est pas étrangère non plus aux polémiques sur la notion de surveillance. Pour nous, il faut substituer au principe d'efficacité un principe de nécessité. Prenons un exemple concret: si vous voulez lutter efficacement contre les violences conjugales, vous pourriez accepter de placer micros et caméras de vidéosurveillance dans tous les domiciles. Dès qu'il se passe quelque chose, on pourrait immédiatement intervenir et ainsi prévenir l'existence de violences conjugales. On voit bien que ce souci d'efficacité conduirait à un abus très clair et une atteinte disproportionnée au droit à l'intimité et aux libertés des personnes. Dans un système démocratique garantissant les libertés individuelles, chaque mesure doit répondre à un principe de nécessité et de proportionnalité. L'efficacité ne peut pas être la seule préoccupation de l'autorité qui met en œuvre la surveillance.

QUESTION : LA LOI A ÉTÉ VOTÉE À UNE LARgE MAJORITÉ DES DÉPUTÉS ET DES SÉNATEURS. APRÈS SA VALIDATION PAR LE CONSEIL CONSTITUTIONNEL SAUF POUR LES DISPOSITIFS CONCERNANT LA SURVEILLANCE INTERNATIONALE, EXISTE-T-IL ENCORE DES RECOURS possibles aU plan National mais aUSSI EUROPÉEN POUR S'OPPOSER À SA MISE EN APPLICATION, EN MODULER LA MISE EN œUVRE EN FONCTION DU NIVEAU DE MENACE VOIRE DE LA RÉVISER APRÈs UN CERTAIN déLAI?

Le texte a été adopté par une très large majorité. Et pourtant, je pense que les organisations très fortement mobilisées contre ce texte ont réussi à imposer dans ce débat leur opposition comme étant légitime - malgré les discours du ministre de l'Intérieur qui visaient à les discréditer par principe - en obtenant que quatre-vingts parlementaires dans chaque assemblée votent contre. Cela parait dérisoire mais c'est malgré tout quelque chose qui a été obtenu de haute lutte, mettant en débat l'ensemble des dérives contenues dans ce texte. Le Conseil constitutionnel a été saisi par ces mêmes parlementaires qui avaient la particularité pour nombre d'entre eux d'être issus 
de la droite et même parfois à l'origine d'un certain nombre de dispositifs antiterroristes ou de surveillance, associés à certains députés de gauche qui n'appartenaient pas à la majorité gouvernementale. La décision du Conseil constitutionnel est dans la droite ligne de sa jurisprudence souvent très soucieuse du droit de propriété mais beaucoup moins sourcilleuse quand il s'agit des libertés individuelles. Concrètement, alors que l'on pouvait penser que le mécanisme de surveillance indifférenciée des réseaux par algorithme pouvait être invalidé par le Conseil constitutionnel comme totalement contraire à un certain nombre de principes fondamentaux, on a une validation qui trouve sa limite dans la surveillance internationale. C'est la seule limite identifiée par le Conseil constitutionnel sur une base assez classique : l'invalidation est procédurale, le législateur n'ayant pas respecté la répartition constitutionnelle entre domaine de la loi et du règlement. Le législateur a abandonné sa compétence au pouvoir exécutif en prévoyant que les modalités du contrôle seraient définies par décret, évidemment secret! La censure du Conseil n'est pas de dire que la surveillance internationale pose problème mais simplement que c'est au législateur de le dire.

La loi sur la surveillance internationale, actuellement en examen, suscite de fortes craintes car nous sommes à nouveau non pas sur une procédure d'urgence mais sur une proposition de loi, laissant croire que c'est un texte initié par des parlementaires. Cette décision du Conseil constitutionnel, n'ayant exercé aucun des contrôles indispensables, risque de laisser cette loi en l'état comme il l'a fait précédemment pour la loi sur le renseignement.

Sur la question de la mise en application, relevons que ces lois de surveillance et de répression antiterroriste obéissent historiquement à un effet de cliquet : il n'y a jamais de retour en arrière sur les dispositifs de surveillance, de fichage. La remise en question de ces dispositifs n'est jamais possible, notamment au nom d'une prétendue efficacité. Pourtant, les effets de ces dispositifs ne sont pas évalués. Avec souvent à l'origine de ces textes un argumentaire qui vise à les faire passer pour une modification temporaire du droit mais qui en réalité s'installe. On identifie dans la succession de ces textes une forme d'extension progressive mais continue de poche " d'exceptionalisme » au sein de notre droit, avec à l'origine de ces textes un argumentaire visant à les faire passer pour une modification temporaire du droit mais qui en définitive s'installe. Ainsi, le droit d'exception ne fonctionne pas comme suspension du droit mais comme développement de pouvoirs exorbitants, de pouvoirs exceptionnels dans un cadre de droit.

L'inquiétude sur le mésusage qui pourrait être fait de ces dispositions existe dans l'hypothèse d'un gouvernement d'extrême-droite mais aussi dès maintenant en réalité. L'action des services de renseignements est placée sous le sceau du principe de précaution, évoqué à de multiples reprises par Bernard Cazeneuve. Les dérives possibles sont déjà contenues dans la loi et peuvent-être mises en œuvre indépendamment de la sensibilité du pouvoir politique

Elles pourraient être décuplées par l'arrivée au pouvoir d'un parti clairement antidémocratique avec des pratiques discriminatoires. Il faut penser la possibilité de dérives dès maintenant sans attendre une situation de changement politique. 


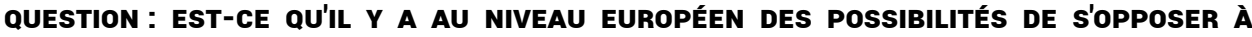 CETTE LOI?}

La Cour européenne des Droits de l'Homme peut-être un recours sur ces mesures de surveillance. Il faut envisager les modalités de saisine de cette juridiction. Des journalistes et des avocats l'ont saisie du texte car il peut mettre en cause directement leur activité professionnelle. La question va être comment faire des recours juridiques pertinents susceptibles d'aboutir à une condamnation de la France sachant que la jurisprudence de la Cour européenne des Droits de l'Homme en matière de surveillance affirme à la fois des grands principes mais a pu valider un certain nombre de dispositifs qui n'étaient pas garantis. On ne peut tout attendre de la Cour européenne des Droits de l'Homme. Ce qui est certain c'est qu'il faut réfléchir notamment à des actions qui pourraient être menées sur la base notamment de situations individuelles et de recours individuel de personnes ayant fait l'objet de mesures de surveillance contestées jusque devant la Cour européenne des Droits de l'Homme. Cela implique d'identifier l'existence de ces abus, ce qui est particulièrement difficile pour deux raisons: on est dans une action secrète par principe avec des conséquences qui sont en apparence indolores; cela reste au niveau de la surveillance tant que cela n'entraîne pas en apparence de conséquences sur les décisions publiques de l'État.

Bien que l'effet cliquet s'oppose au retour en arrière, le combat doit continuer à être mené au plan politique.

\section{QUESTION : VOS POSITIONS SONT-ELLES PARTAgÉES PAR TOUS LES MAgISTRATS ? CE DÉbAT ENTRE-T-IL DANS LA FORMATION DES FUTURS MAgISTRATS ?}

Sur ce texte, il y a eu une opposition assez générale des magistrats qui ont identifié les risques de dérive car en tant qu'autorité de contrôle des services de police ayant aussi pour mission de garantir les libertés individuelles, on identifie les possibilités de dérive dans un système de droit pénal. L'Union syndicale des magistrats s'est également opposée à la loi même si ce syndicat n'a pas pris une part active au mouvement ; bien que favorables aux lois précédentes, certains juges d'instruction antiterroristes y ont également vu une mutation du système institutionnel. Toutefois, l'analyse d'une extension de l'exception et le sentiment qu'il n'y a pas de retour en arrière possible en la matière sont un peu moins partagés par l'ensemble de la magistrature. Sur la formation des magistrats, la garantie des libertés est présente mais elle est rongée de l'intérieur par un discours politique répressif qui affirme que doit primer une prétendue efficacité. Le milieu de la magistrature n'est pas indemne de ces évolutions sociétales et de l'envahissement du débat par des conceptions comme "la sécurité serait la première des libertés ", martelées par la droite et reprises par la gauche à la fin des années 90. Il faut y résister en rappelant le nécessaire contrôle de toutes les atteintes aux libertés individuelles et, comme l'a affirmé la Cour européenne des Droits de l'Homme, veiller à ne pas saper les fondements de la démocratie au prétexte de la défendre. 


\section{NOTES}

1. Rapport $n^{\circ} 1056$ de l'Assemblée nationale sur le fonctionnement des services de renseignements français dans le suivi et la surveillance des mouvements radicaux armés, Christophe Cavard et Jean-Jacques Urvoas, mai 2013, http://scsi-pn.fr/wp-content/uploads/2013/05/ RAPPORT_CAVARD_URVOAS.pdf

2. Le 17 octobre 2011, alors qu'il est directeur central du renseignement intérieur, Bernard Squarcini est mis en examen par la juge Sylvia Zimmermann pour «atteinte au secret des correspondances », " collecte illicite de données » et " recel du secret professionnel », dans le cadre de l'«affaire des fadettes» Source: Le Monde du 18/10/2011, http://www.lemonde.fr/ societe/article/2011/10/18/fadettes-ce-que-bernard-squarcini-a-dit-a-la-

juge_1589540_3224.html.

3. La Commission nationale de contrôle des interceptions de sécurité (CNCIS) est une autorité administrative française instituée par la loi du 10 juillet 1991 dont la mission est la vérification de la légalité des autorisations d'interception (écoutes téléphoniques non-judiciaires), à compter de 2003 aux interceptions demandées en urgence absolue. Jean-Marie Delarue présidait cette institution depuis 2014 mais a été écarté de ce poste par le gouvernement Valls le 15 septembre 2015.

4. fausses antennes relai qui peuvent capter toutes les données téléphoniques sur un rayon qui va de quelques centaines de mètres jusqu'à deux kilomètres

\section{RÉSUMÉS}

Bien que cette longue interview de la secrétaire générale du Syndicat de la Magistrature date d'octobre 2015, il nous semble nécessaire de la publier tant le vote de la loi sur le renseignement suite aux attentats du 11 janvier 2015 ouvre une période sombre pour ce qui reste d'institutions démocratiques à la société française. En effet, cette loi sur le renseignement institue une mise en surveillance totale de la société, concédant des pouvoirs exorbitants de contrôle de l'espace privé (poses de micros, IMSI catcher, captation de données informatiques archivées ..) et de fouille dans l'espace public tout en affaiblissant les droits fondamentaux du justiciable. Cette loi est le socle juridique d'une politique du "tout sécuritaire» dont il faut craindre qu'elle ne serve d'abord les ennemis d'une société démocratique ouverte à l'altérité. Depuis d'autres textes se sont ajoutés à l'arsenal sécuritaire confirmant le recul du droit devant les illusions d'une société à la recherche comme souvent dans les périodes difficiles de son histoire, d'un homme (ou d'une femme) providentiel.

\section{INDEX}

Mots-clés : Loi sur le renseignement, syndicat de la magistrature 
AUTEURS

DOMINIQUE DESBOIS

JACQUES VÉTOIS 